\title{
Efecto hepatoprotector de yacón (Smallanthus sonchifolius) en ratas tratadas con acetaminofén: biomarcadores hepáticos de estrés oxidativo
}

Inés Arnao, Silvia Suárez, Juan Trabucco, Ruth Cisneros, Raquel Oré, Rubén Valdivieso, Rosa Oriondo

Centro de Investigación de Bioquímica y Nutrición, Facultad de Medicina, UNMSM

Objetivos: Evaluar el efecto hepatoprotector del extracto acuoso de hojas de yacón (EHY) en ratas intoxicadas con acetaminofén, mediante biomarcadores de estrés oxidativo.

Diseño: Analítico, experimental.

Institución: Centro de Investigación de Bioquímica y Nutrición, Facultad de Medicina, UNMSM.

Material biológico: EHY, obtenido de Yauyos-Lima.

Intervenciones: Se formó 4 grupos de ratas hembras $(n=6)$. Se les administró por vía oral lo siguiente; G1: control (suero fisiológico (SF)) por 11 días; G2, G3 y G4: paracetamol 300 mg/kg por 6 días y, luego, por 5 días SF, EHY, $400 \mathrm{mg} / \mathrm{kg}$ y silimarina $50 \mathrm{mg} / \mathrm{kg}$, respectivamente.

Principales medidas de los resultados: Lipoperoxidación, superóxido-dismutasa (SOD), catalasa (CAT), glutatión peroxidasa y glutatión S-transferasa.

Resultados: Los niveles de lipoperoxidación de G3 (6,5 umol/L) fueron menores que G1 (7,9 umol/L). La relación de enzimas antioxidantes SOD /CAT fue 1,84, en G3 mayor a 1,24 de G2. La glutatión peroxidasa en G3 mostró una disminución de $63 \%$ frente a G2 y la actividad de glutatión S-transferasa incrementó en $52 \%$ frente a este mismo grupo.

Conclusiones: El EHY mostró un leve efecto hepatoprotector a través de los indicadores antioxidantes relacionados con las especies reactivas del oxigeno (EROs) y mediante el indicador de detoxificación de fase II, lo que produjo una ligera disminución de los niveles de lipoperoxidación.

Palabras clave: Hepatotoxicidad, acetaminofén, Smallanthus sonchifolius, SOD, catalasa, lipoperoxidación.

\section{Capacidad antioxidante in vitro y contenido de polifenoles y flavonoides en el extracto hidroalcóholico de las hojas de Trixis divaricata (hank'u chuta)}

Gloria Mayhua, Silvia Suárez, Tatiana del Castillo

Centro de Investigación de Bioquímica y Nutrición, Facultad de Medicina, UNMSM; Carrera de Farmacia y Bioquímica, UNSAC

Objetivos: Evaluar la capacidad antioxidante in vitro y el contenido de polifenoles y flavonoides en un extracto seco hidroalcohólico de las hojas de Trixis divaricata (H.B.K.) Sprengel (EHAH).

Diseño: Descriptivo.

Institución: Centro de Investigación de Bioquímica y Nutrición, Facultad de Medicina, UNMSM; Carrera de Farmacia y Bioquímica, UNSAC.

Material biológico: EHAH, obtenido de Yarkapata, Cusco.

Intervenciones: Se preparó un extracto hidroalcohólico 70\%. Se determinó humedad y solubilidad. Para la determinación antioxidante in vitro en medio biológico, se preparó homogenizado de hígado de rata al 10\%.

Principales medidas de los resultados: Porcentaje de humedad; capacidad antioxidante: porcentaje de captación de $\mathrm{DPPH}, \mathrm{IC} 50(\mathrm{ug} / \mathrm{mL}$ ) y MDA (nmol/g tejido); polifenoles (mg AG/g mp) y flavonoides (mg Q/g mp); ácido gálico (AG), quercetina $(\mathrm{Q})$.

Resultados: Se presentó $49 \%$ de humedad y fue soluble en metanol. Polifenoles: 273,4. Flavonoides: 95,2. Porcentaje de captación de DPPH fue 72,6\% a una concentración de $12 \mathrm{ug} / \mathrm{mL}, \mathrm{IC} 50: 7,92 \mathrm{ug} / \mathrm{mL}$. El estándar vitamina C tuvo un IC 50: 2,04 ug/mL. La concentración de MDA fue 1,8 nmol/g tejido a una concentración de $600 \mathrm{ug} / \mathrm{mL}$ extracto; el estándar de referencia fue vitamina $\mathrm{E}$ y tuvo $1,4 \mathrm{nmol} \mathrm{MDA} / \mathrm{g}$ tejido a una concentración de $20 \mathrm{ug} / \mathrm{mL}$.

Conclusiones: El EHAH mostró capacidad antioxidante in vitro, probablemente por su contenido y calidad de metabolitos secundarios.

Palabras clave: Trixis divaricata (HBK) Sprengel, antioxidante, radical libre, flavonoide. 